\title{
Estrutura da ictiofauna do rio Bonito, bacia hidrográfica do rio Ivaí, sistema alto rio Paraná, Brasil
}

\author{
Douglas Viana ${ }^{1,5}$, Cláudio Henrique Zawadzki ${ }^{1,2}$, Edson Fontes de Oliveira ${ }^{3}$, \\ Huilquer Francisco Vogel $^{4}$ \& Weferson Júnio da Graça ${ }^{2}$

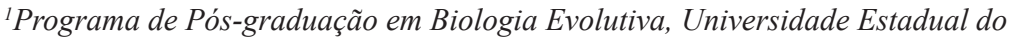 \\ Centro-Oeste - UNICENTRO, Rua Simeão Camargo Varela de Sá, 03, \\ Bairro Cascavel, CEP 85040-080, Guarapuava, PR, Brasil \\ http://www.unicentro.br/ \\ ${ }^{2}$ Núcleo de Pesquisas em Limnologia, Ictiologia e Aquicultura - NUPÉLIA \\ Departamento de Biologia, Universidade Estadual de Maringá - UEM, \\ Av. Colombo, 5790, CEP 87020-900, Maringá, PR, Brasil. http://www.nupelia.uem.br/ \\ ${ }^{3}$ Departamento de Engenharia Ambiental, Universidade Tecnológica Federal do Paraná - UTFPR, \\ Av. dos Pioneiros, 3131, Jardim Marumbi, CEP 86036-370, Londrina, PR, Brasil \\ http://www.utfpr.edu.br/londrina \\ ${ }^{4}$ Programa de Pós-graduação em Ecologia de Ambientes Aquáticos Continentais, Universidade Estadual de \\ Maringá - UEM, Av. Colombo, 5790, CEP 87020-900, Maringá, PR, Brasil. http://www.pea.uem.br/ \\ 5Autor para correspondência: Douglas Viana,e-mail:douglasvbr@ibest.com.br
}

VIANA, D., ZAWADZKI, C.H., OLIVEIRA, E.F., VOGEL, H.F. \& GRAÇA, W.J. Structure of the ichthyofauna of the Bonito river, Ivaí river basin, upper Paraná river system, Brazil. Biota Neotrop. (13)2: http://www. biotaneotropica.org.br/v13n2/en/abstract?inventory+bn03013022013

\begin{abstract}
The ichthyofauna diversity of the Ivaí river basin is largely underestimated. The present study aimed to characterize the fish fauna of the Bonito River, a tributary to the Ivaí River, upper Paraná river basin, in relation to richness, diversity and constancy. It was established three sampling sites along the longitudinal gradient of the river: headwater, medium stretch and mouth, at the point near the confluence to Ivaí river. Three collection expeditions were made in each site. Gill nets of different meshes, cast nets and traps were used. The ichthyofauna of the Bonito river was composed by 23 species, divided into nine families and three orders. Among the sampled species, three were possible new records for the upper Paraná river basin and 12 for the Ivaí river basin. The highest species richness was recorded in the lower stretch, where 20 species were found. In the middle section of the river, seven species were collected and just one species was captured at headwater. In the lower stretch it was also obtained the greatest diversity and lower species dominance. Additionally, in the lower Bonito river most of the species were incidental, whereas in the middle section five species were constant and only one was accessory. The middle section had the most dominant species, Hypostomus aff. ancistroides. The altitudinal gradient seems to determine the pattern of species distribution, delimiting the assemblages along longitudinal gradient of the river. Keywords: diversity, fishes, freshwater, neotropical region, streams.
\end{abstract}

VIANA, D., ZAWADZKI, C.H., OLIVEIRA, E.F., VOGEL, H.F. \& GRAÇA, W.J. Estrutura da ictiofauna do rio Bonito, bacia hidrográfica do rio Ivaí, sistema alto rio Paraná, Brasil. Biota Neotrop. 13(2): http://www. biotaneotropica.org.br/v13n2/pt/abstract?inventory+bn03013022013

Resumo: A diversidade da ictiofauna da bacia do rio Ivaí é altamente subestimada. O presente estudo teve como objetivo caracterizar a ictiofauna do rio Bonito, um tributário do rio Ivaí, bacia do alto rio Paraná, quanto à riqueza, diversidade e constância de espécies. Foram estabelecidos três locais de amostragem ao longo do gradiente longitudinal do rio: cabeceira, trecho médio e região de desembocadura próxima ao rio Ivaí. Três incursões de coleta foram feitas em cada unidade utilizando redes de espera de diferentes malhas, tarrafas e covos. A ictiofauna foi composta por 23 espécies, divididas em nove famílias e três ordens. Entre as espécies coletadas, três delas são possíveis novos registros para o alto rio Paraná e 12 para a bacia do rio Ivaí. A maior riqueza foi registrada no trecho inferior, onde foram encontradas 20 espécies, o qual também apresentou maior diversidade e menor dominância de espécies. No trecho médio do rio foram coletadas sete espécies e no trecho superior apenas uma. No baixo rio Bonito a maioria das espécies foram acessórias e no trecho médio cinco foram constantes e apenas uma acessória. O trecho médio apresentou maior dominância de espécies, com destaque para Hypostomus aff. ancistroides. O gradiente altitudinal foi um importante fator estruturador da assembleia de peixes do rio Bonito, delimitando as assembleias ao longo do gradiente longitudinal.

Palavras-chave: diversidade, peixes, água doce, região neotropical, riachos. 


\section{Introdução}

A região neotropical apresenta uma ictiofauna diversificada e está entre as mais numerosas do planeta. Nos últimos séculos, a sua complexidade ictiofaunística tem sido constatada por vários autores, dentre os quais podemos destacar os trabalhos de Eigenmann \& Eigenmann (1891), cujos inventários estimaram a fauna de peixes neotropicais em 1.297 espécies. Posteriormente, Géry (1969) estimou para esta região entre 2.400 a 2.700 espécies de peixes. Dados mais recentes são bastante divergentes, variando entre 2.400 e 4.000 para Mcallister et al. (1997), 4.475 segundo Reis et al. (2003) e até mesmo dados estimados em cerca de 8.000 espécies (Schaefer 1998).

No Brasil, uma importante compilação de dados foi realizada por Fowler (1948), o qual registrou pouco mais de 1.000 espécies. Buckup et al. (2007) realizaram o último levantamento geral e estimaram 2.481 espécies de peixes de água doce no Brasil. Grande parte dessa diversidade está na bacia do rio Paraná, que apresenta cerca de 600 espécies conhecidas (Pelicice et al. 2005). O sistema do alto rio Paraná está entre as bacias melhor estudadas no território brasileiro, constituindo uma área com uma ictiofauna complexa e singular, onde, no entanto, nota-se que ainda é grande o número de rios não inventariados.

Segundo Langeani et al. (2007) a bacia do alto rio Paraná abriga 310 espécies de peixes, distribuídas em 11 ordens e 38 famílias, com elevado potencial para o registro de novas espécies, principalmente em ambientes de riachos. A composição e número de espécies de peixes conhecidas vêm crescendo com o aumento dos esforços de coleta e descrição de novas espécies.

A maioria dos trabalhos sobre a ictiofauna neotropical referese à ambientes fluviais de grande porte, mas cerca de 50\% dessa diversidade é composta por espécies pequenas, provenientes de pequenos rios ou riachos. Estas espécies apresentam elevado grau de endemismo e ocupam uma grande variedade de microhabitats específicos, sendo geralmente menos estudadas que espécies de maior tamanho. Castro (1999) argumenta que um importante desafio para a ictiologia sul-americana consiste no estudo da sistemática, evolução e biologia de peixes de água doce de pequeno porte.

A complexidade da ictiofauna de riachos é comumente estruturada pelo gradiente altitudinal, sendo que aqueles riachos que apresentam maior variação em altitude possuem ictiofauna reduzida em relação àqueles com gradiente menor (Buckup 1999). De modo geral, a fauna de peixes de riachos forma um conjunto pouco conhecido. Entre as 52 espécies coletadas por Castro et al. (2003) em trechos de 17 riachos do Paranapanema, $25 \%$ destas eram pouco ou não conhecidas, sendo oito delas seguramente novas e cinco com status taxonômico indefinido. Araújo \& Tejerina-Garro (2007), em riachos de cerrado da bacia do ribeirão Ouvidor no alto Paraná, registraram uma riqueza de 35 espécies sendo que $40 \%$ destas foram identificadas apenas ao nível de gênero, demonstrando a necessidade de estudos taxonômicos da ictiofauna do rio Paraná.

Dentre todas as bacias hidrográficas que fazem parte do sistema alto rio Paraná, a do rio Ivaí é uma das menos estudadas, revelando uma ictiofauna praticamente desconhecida (Galves et al. 2009). Maier et al. (2008) realizaram um inventário da ictiofauna no rio Barra Bonita, um tributário do rio Ivaí. Neste estudo foram listadas 31 espécies pertencentes a cinco ordens, 14 famílias e 25 gêneros. Entre as espécies capturadas, as três mais representativas podem tratar-se de espécies novas. Estes resultados demonstram a importância da realização de estudos e levantamentos mais amplos da ictiofauna no sistema alto rio Paraná, sobretudo da bacia do rio Ivaí. Neste contexto, o presente estudo investigou a estrutura da ictiofauna do rio Bonito, afluente do rio Ivaí, avaliando os padrões de riqueza, diversidade e constância.

\section{Material e Métodos}

A área de estudo compreende o rio Bonito, afluente do rio Ivaí, sistema do Alto rio Paraná (Figura 1). O rio Bonito delimita os municípios paranaenses de Turvo e Boa Ventura de São Roque, ao longo de um gradiente longitudinal de $120 \mathrm{~km}$ de extensão.

O clima predominante é o subtropical úmido mesotérmico de temperatura média anual de $20^{\circ} \mathrm{C}$, com precipitação média anual de 1.600 a $1.700 \mathrm{~mm}$. O rio Bonito está inserido nas unidades geológicas de formação da Serra Geral, Pirambóia, Botucatu e Formação Rio do Rastro. Seu curso corre no sentido leste do município de Turvo e desemboca ao norte, na margem esquerda do rio Ivaí (Viana et al. 2008).

O rio Bonito apresenta na maior parte de seu curso ambientes de correnteza rápida a moderada, com profundidade média próxima a $1,5 \mathrm{~m}$, alternado por remansos e poções com profundidades um pouco superiores. O substrato do rio é rochoso, com pouca deposição de sedimentos, na maior parte constituída de galhos, pequenos troncos e matéria particulada, predominantemente retidos nas margens.

Uma das características deste rio é o desnível observado entre os trechos superior $(1.002 \mathrm{~m})$, médio $(790 \mathrm{~m})$ e inferior $(461 \mathrm{~m})$, com presença de quedas de água durante seu percurso, sendo uma delas localizada entre os trechos inferior e médio, com altura superior a 80 m. Com relação ao uso e ocupação das margens, na região da cabeceira a principal atividade é a agricultura, que tem contribuído para a destruição da vegetação ripária e o assoreamento do rio. É evidente a instabilidade das margens com muitas áreas descobertas, com erosão transportando grandes quantidades de sedimento das áreas de cultivo que se depositam no leito do rio. Os principais tipos de ocupação nos trechos médio e inferior são a pecuária e a agricultura. O trecho médio apresenta uma elevada diversidade de habitats, no entanto é notável o processo de degradação da mata ciliar. $\mathrm{O}$ baixo rio Bonito tem relevo com encostas íngremes e vale mais profundo, apresenta corredeiras bem desenvolvidas, alternadas por remansos, com presença de macrófitas nas margens. Tanto o trecho baixo como o médio possuem substrato de fundo rochoso, formado principalmente por pedras e cascalho, que favorecem a proliferação do perifíton.

Foram estabelecidas três locais de amostragens ao longo do curso do rio, sendo uma na cabeceira $25^{\circ} 00^{\prime} 34,57^{\prime \prime} \mathrm{S}$ e $51^{\circ} 39^{\prime}$ $27,74^{\prime \prime}$ O, uma no curso médio $24^{\circ} 52^{\prime} 16,96^{\prime \prime} \mathrm{S}$ e $51^{\circ} 26^{\prime} 56,48^{\prime \prime}$ $\mathrm{O}$ e outra na região de desembocadura no rio Ivaí $24^{\circ} 46^{\prime} 44,53^{\prime \prime} \mathrm{S}$ e $51^{\circ} 25^{\prime} 35,39$ " O (Figura 1). Foram realizadas três incursões de coleta em cada local: 29/01/09 a 02/02/09; 23/02/09 a 25/02/09; e $23 / 05 / 09$ a 25/05/09. Neste trabalho não foi possível a realização de amostragens periódicas, buscando investigar influências sazonais, devido à ocorrência de chuvas excessivas ao longo do ano de 2009 na região da bacia do rio Bonito, o que impediu o acesso aos locais de amostragem.

Nas coletas foram utilizadas redes de espera medindo $1,5 \mathrm{~m}$ de altura por $20 \mathrm{~m}$ de comprimento com malhas $1,5 \mathrm{~cm}, 3 \mathrm{~cm}, 4 \mathrm{~cm}$ e $5 \mathrm{~cm}$ entre nós opostos, bem como tarrafa com malha $1,2 \mathrm{~cm}$ entre nós opostos, peneiras e dois covos de plástico, medindo $1 \mathrm{~m}$ de comprimento por $40 \mathrm{~cm}$ de diâmetro. As redes de espera, assim como os covos, foram instalados ao final de tarde e retirados no início da manhã do dia seguinte, permanecendo por um período de 16 horas nos locais de amostragem. Utilizou-se ração comercial para peixe envolvida por tecido, como isca nos covos, a fim de evitar que os espécimes ingerissem a isca. Para coletas através de peneiras e tarrafa, foi estabelecido um trecho de cerca de $300 \mathrm{~m}$ de extensão em cada local de amostragem, onde foram efetuadas 100 batidas pelo período de aproximadamente uma hora em cada período amostral. Os indivíduos coletados foram anestesiados com cloridrato de benzocaína $10 \%$ e imediatamente acondicionados em recipientes de plástico, 




Figura 1. Representação esquemática das unidades amostrais (trechos alto, médio, e baixo) situadas no rio Bonito, afluente da margem esquerda do rio Ivaí, Estado do Paraná.

Figure 1. Representation of the collecting points (upper stretches, medium, and lower) in the Bonito river, tributary of the left margin of the Ivaí river, State of Paraná.

contendo formol a $4 \%$, etiquetados e identificados por localidade. No laboratório foram tomados os seguintes dados biométricos: peso total (PT, em gramas) e comprimento padrão (CP, em centímetros). Os espécimes foram identificados segundo Graça \& Pavanelli (2007). Indivíduos testemunho das espécies coletadas foram depositados na coleção ictiológica do Núcleo de Pesquisas em Limnologia, Ictiologia e Aquicultura (NUP) da Universidade Estadual de Maringá (UEM), disponível em http://peixe.nupelia.uem.br/ (verificar apêndice).

Os padrões de diversidade e dominância foram estimados a partir do índice de Shannon (Ludwig \& Reynolds 1988) e índice de Simpson, respectivamente (Magurran 1988). A hipótese nula de que não há diferença significativa entre os índices de diversidade dos diferentes locais foi testada aplicando a adaptação do teste "t" de Student proposto Hutcheson (1970) (ver Zar 1999). A constância das espécies foi calculada para cada ponto de coleta, de acordo com o critério recomendado por Dajoz (1983): espécies com frequência de ocorrência em até $25 \%$ foram classificadas como acidentais, entre $25 \%$ e $50 \%$ como acessórias, e acima dos $50 \%$ como constantes. Para os ambientes amostrados foi calculado o índice de similaridade de Jaccard, pela expressão $C J=C /(A+B-C)$, na qual $C J=$ índice de similaridade Jaccard, $A$ = número de espécies encontradas no local $A, B=$ número de espécies encontradas no local $B$, e $C=$ número de espécies comuns aos locais $A$ e $B$ (Magurran 1988).

\section{Resultados}

No rio Bonito foram coletados 392 indivíduos, distribuídos em 23 espécies, nove famílias e três ordens. $\mathrm{O}$ trecho baixo do rio Bonito registrou a maior riqueza de espécies (20 espécies), seguido pelos trechos médio (sete) e superior (uma) (Tabela 1).

Dentre as ordens, as que apresentaram maiores riquezas foram Characiformes com 12 espécies e Siluriformes com 10 espécies, ambas com quatro famílias, seguidas por Perciformes com uma espécie. Siluriformes e Characiformes registraram as maiores abundâncias, com 257 e 120 indivíduos, respectivamente, enquanto da ordem Perciformes foram capturados 15 espécimes (Tabela 1).

As famílias mais representativas nos ambientes amostrados foram Loricariidae, com cerca de $75 \%$ dos espécimes coletados no trecho médio do rio e $50 \%$ no trecho baixo, enquanto Characidae teve um percentual de $15 \%$ no trecho médio e $20 \%$ no baixo. Parodontidae contribuiu com cerca de $10 \%$ dos espécimes coletados no médio e baixo trechos do rio (Tabela 1). No alto rio Bonito, Astyanax aff. paranae (Characidae) foi a única espécie capturada, revelando-se comum entre os trechos alto e médio. Entre os trechos médio e baixo foram registradas quatro espécies comuns: Apareiodon vladii, Astyanax aff. fasciatus, Geophagus brasiliensis e Rhamdia quelen. $\mathrm{O}$ trecho médio, apesar do número intermediário de espécies, apresentou maior abundância $(n=290)$ em relação ao trecho baixo $(n=58)$ e alto $(n=44)$ (Tabela 1$)$.

As espécies mais representativas no rio Bonito foram Hypostomus aff. ancistroides, do curso médio, com 207 exemplares (52,8\%); seguida por Astyanax aff. paranae, dos trechos alto e médio (59 exemplares, 15,0\%), e Astyanax aff. fasciatus dos trechos médio e baixo, onde foram capturados 34 espécimes (8,7\%; Figura 2). Por outro lado, as espécies que mais contribuíram em biomassa foram: 
Hypostomus aff. ancistroides (61,8\%), Rhamdia quelen $(8,7 \%)$, Hypostomus sp. 1 (8,1\%) e Astyanax aff. paranae (3,8\%) (Figura 2).

Quanto à presença de espécies, 18 delas foram coletadas em apenas um único trecho (duas no médio e 16 no baixo rio Bonito), enquanto cinco foram capturadas em pelo menos dois trechos (Apareiodon vladii, Astyanax aff. fasciatus, Geophagus brasiliensis, Rhamdia quelen (no médio e baixo) e Astyanax aff. paranae (presente no alto e médio rio Bonito). Nenhuma espécie teve ocorrência nos três trechos amostrados (Tabela 1).
Dentre as espécies coletadas, 11 foram consideradas constantes, sendo seis no trecho médio, quatro no baixo e uma espécie no alto rio Bonito, enquanto 17 foram consideradas acessórias, 16 destas no trecho inferior e uma no médio. Astyanax aff. paranae foi a única espécie constante em dois pontos de coleta, no alto e médio rio Bonito (Tabela 1).

No conjunto de espécies coletadas no rio Bonito, possivelmente, quatro delas ainda são desconhecidas da ciência: Astyanax sp., Hypostomus sp. 1, Hypostomus sp. 2, e Trichomycterus sp. Dentre as espécies, três delas ainda possuem status taxonômico indefinido,

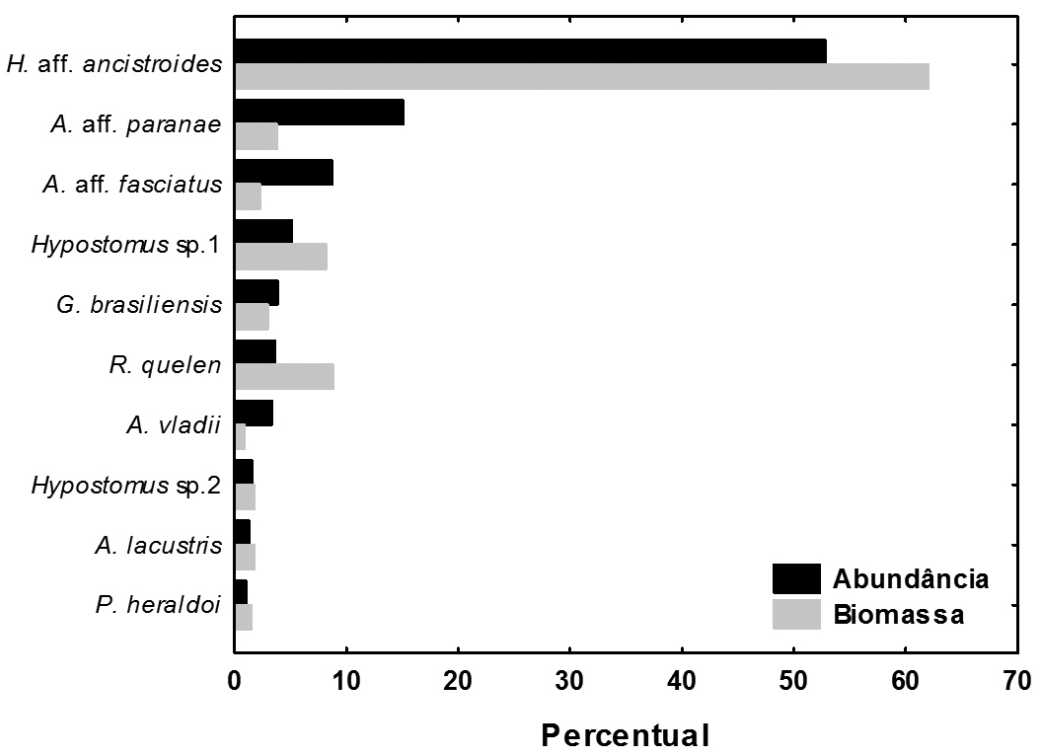

Figura 2. Abundância (barras negras) e biomassa (barras cinzas) das principais especies de peixes coletadas no rio Bonito, afluente da margem esquerda do rio Ivaí, Estado do Paraná.

Figure 2. Abundance (black bars) and biomass (grey bars) from the main fish species collected in the Bonito river, tributary of the left margin of the Ivaí river, State of Paraná.

Tabela 1. Posição taxonômica das espécies, abundância total $\left(\mathrm{N}_{\text {total }}\right)$, e espécies constantes $(\mathrm{Co})$, acessórias (Ace) e acidentais (Aci) para os trechos alto, médio e baixo do rio Bonito, bacia do rio Ivaí, Paraná, Brasil.

Table 1. Species taxonomic ranking, total abundance $\left(\mathrm{N}_{\text {Total }}\right)$, and constancy $(\mathrm{Co})$, accessory (Ace) and accidental (Aci) species to the sites upper, medium, and lower Bonito River, Ivaí River basin, State of Paraná, Brazil.

\begin{tabular}{|c|c|c|c|c|c|c|c|c|c|c|c|c|}
\hline \multirow{2}{*}{ Táxon } & \multicolumn{4}{|c|}{ Alto Bonito } & \multicolumn{4}{|c|}{ Médio Bonito } & \multicolumn{4}{|c|}{ Baixo Bonito } \\
\hline & $\mathbf{N}_{\text {Total }}$ & Co & Ace & Aci & $\mathbf{N}_{\text {Total }}$ & Co & Ace & Aci & $\mathbf{N}_{\text {Total }}$ & Co & Ace & Aci \\
\hline \multicolumn{13}{|l|}{ Characiformes } \\
\hline \multicolumn{13}{|l|}{ Characidae } \\
\hline Astyanax aff. fasciatus (Cuvier, 1819) & - & & & & 30 & + & & & 4 & & & \\
\hline Astyanax aff. paranae (Eigenmann, 1914) & 44 & + & & & 15 & + & & & - & & & \\
\hline Astyanax altiparanae Garutti \& Britski, 2000 & - & & & & 2 & & + & & - & & & \\
\hline Astyanax sp. & - & & & & - & & & & 1 & & + & \\
\hline Oligosarcus paranensis Menezes \& Géry, 1983 & - & & & & - & & & & 1 & & + & \\
\hline Brycon nattereri (Günther, 1864) & - & & & & - & & & & 1 & & + & \\
\hline Galeocharax knerii (Steindachner, 1879) & - & & & & - & & & & 1 & & + & \\
\hline \multicolumn{13}{|l|}{ Parodontidae } \\
\hline Apareiodon vladii Pavanelli, 2006 & - & & & & 9 & + & & & 4 & & + & \\
\hline Apareiodon affinis Steindachner, 1879 & - & & & & - & & & & 1 & & + & \\
\hline \multicolumn{13}{|l|}{ Acestrorhynchidae } \\
\hline Acestrorhychus lacustris Lütken, 1875 & - & & & & - & & & & 5 & + & & \\
\hline \multicolumn{13}{|l|}{ Anostomidae } \\
\hline Leporinus octofasciatus Steindachner, 1915 & - & & & & - & & & & 2 & & + & \\
\hline Leporinus amblyrhynchus Garavello \& Britski, 1987 & - & & & & - & & & & 1 & & + & \\
\hline
\end{tabular}


Tabela 1. Continuação...

\begin{tabular}{|c|c|c|c|c|c|c|c|c|c|c|c|c|}
\hline \multirow{2}{*}{$\begin{array}{cc}\text { Táxon } \\
\end{array}$} & \multicolumn{4}{|c|}{ Alto Bonito } & \multicolumn{4}{|c|}{ Médio Bonito } & \multicolumn{4}{|c|}{ Baixo Bonito } \\
\hline & $\mathbf{N}_{\text {Total }}$ & Co & Ace & Aci & $\mathbf{N}_{\text {Total }}$ & Co & Ace & Aci & $\mathbf{N}_{\text {Total }}$ & Co & Ace & Aci \\
\hline \multicolumn{13}{|l|}{ Siluriformes } \\
\hline \multicolumn{13}{|l|}{ Loricariidae } \\
\hline Hypostomus ancistroides (Ihering, 1911) & - & & & & - & & & & 1 & & + & \\
\hline Hypostomus aff. ancistroides (Ihering, 1911) & - & & & & 207 & + & & & - & & & \\
\hline Hypostomus albopunctatus (Regan, 1908) & - & & & & - & & & & 1 & & + & \\
\hline Hypostomus sp. 1 & - & & & & - & & & & 20 & + & & \\
\hline Hypostomus sp. 2 & - & & & & - & & & & 6 & + & & \\
\hline \multicolumn{13}{|l|}{ Pimelodidae } \\
\hline Pimelodus ornatus Kner, 1858 & - & & & & - & & & & 1 & & + & \\
\hline Pimelodus microstoma Steindachner, 1877 & - & & & & - & & & & 4 & & + & \\
\hline \multicolumn{13}{|l|}{ Heptapteridae } \\
\hline Imparfinis borodini Mees \& Cala, 1989 & - & & & & - & & & & 1 & & + & \\
\hline Rhamdia quelen (Quoy \& Gaimard, 1824) & - & & & & 13 & + & & & 1 & & + & \\
\hline \multicolumn{13}{|l|}{ Trichomycteridae } \\
\hline Trichomycterus sp. & - & & & & - & & & & 1 & & + & \\
\hline \multicolumn{13}{|l|}{ Perciformes } \\
\hline \multicolumn{13}{|l|}{ Cichlidae } \\
\hline Geophagus brasiliensis (Quoy \& Gaimard, 1824) & - & & & & 14 & + & & & 1 & & + & \\
\hline Total & 44 & & & & 290 & & & & 58 & & & \\
\hline
\end{tabular}

possivelmente tratando-se de novas espécies (Hypostomus aff. ancistroides, Astyanax aff. fasciatus e Astyanax aff. paranae).

Os trechos médio e baixo do rio Bonito revelaram diferença significativa nos valores do índice de diversidade de Shannon $\left(\mathrm{t}=-3,8098 ; \mathrm{t}_{0,05(2) ;} 63,36=1,998 ; \mathrm{P}<0,05 ; \mathrm{H}_{\text {trecho médio }}^{\prime}=0,4586\right.$; $\left.\mathrm{H}_{\text {trecho baixo }}^{\prime}=1,0390\right)$. O índice de Simpson demonstrou que o médio rio Bonito possui maior dominância de espécies em relação ao trecho baixo ( 0,53 e 0,16 , respectivamente; Tabela 1$)$. As curvas espécie-abundância revelam um padrão de maior dominância para o trecho médio do rio Bonito, com contribuição significativa de $H$. aff. ancistroides. Por outro lado, a curva representativa do trecho baixo indica um padrão com maior uniformidade na distribuição das espécies, com elevada proporção de espécies raras (Figura 3). $\mathrm{O}$ alto rio Bonito apresentou uma única espécie, por isso não foi possível avaliar seus padrões de dominância e diversidade. $\mathrm{O}$ índice de similaridade de Jaccard demonstrou pouca semelhança entre os trechos médio e baixo $(0,25)$, correspondendo a 0,41 para o trecho médio e 0,54 para o trecho baixo do rio Bonito.

\section{Discussão}

Dentre as 23 espécies registradas para o rio Bonito, 15 foram citadas por Langeani et al. (2007) entre as 310 espécies da ictiofauna do alto rio Paraná. Maier et al. (2008) listaram para o rio Barra Bonita, também afluente do rio Ivaí, 31 espécies, dentre as quais, dez delas foram comuns ao rio Bonito (Acestrorhynchus lacustris, Apareiodon affinis, Astyanax aff. fasciatus, Astyanax aff. paranae, A. altiparanae, Oligosarcus paranensis, Hypostomus aff. ancistroides, Leporinus amblyrhynchus, Rhamdia quelen e Geophagus brasiliensis). Araújo et al. (2011) encontraram 20 espécies em um riacho de primeira ordem da bacia do rio Ivaí, das quais quatro (Astyanax aff. fasciatus, Astyanax aff. paranae, Oligosarcus paranensis, e Hypostomus ancistroides) também ocorreram no rio Bonito. Desta forma, considerando os levantamentos realizados por Langeani et al. (2007), Maier et al. (2008), e Araújo et al. (2011), 12 espécies registradas nesse trabalho constituem novos registros para a bacia do rio Ivaí e três possíveis para o Alto Paraná (Astyanax sp., Hypostomus sp. 1, Hypostomus sp. 2).

A ictiofauna do rio Bonito está distribuída seguindo um padrão, tanto de aumento no número de espécies, quanto de substituição de espécies do trecho superior para o inferior. A estruturação de comunidades em gradientes de diversidade tem sido apontada por diversos autores, tais como Teixeira et al. (2005), Súarez \& Petrere Júnior (2006), Araújo \& Tejerina-Garro (2007, 2009). De acordo com Vanotte et al. (1980), o conceito de rio contínuo prevê a distribuição das espécies seguindo um gradiente abiótico longitudinal, sob o qual a riqueza e diversidade de espécies tendem a aumentar da cabeceira em direção à foz. Neste padrão, a disponibilidade e complexidade de micro habitats crescem em função do aumento da ordem e vazão dos rios, o que promove incremento de espécies.

O potencial de dispersão das espécies de peixes constitui um importante fator na distribuição geográfica das espécies, o qual varia entre os diferentes grupos taxonômicos. Segundo Lundberg (1993), a dispersão dos peixes de água doce depende de conexões diretas entre bacias hidrográficas e a ocorrência dessas interconexões reflete a subjacente história geológica das paisagens, permitindo o estabelecimento de inferências seguras sobre a evolução física e biótica de uma região. Em peixes, processos geológicos que ligam e separam diferentes sistemas de drenagem, influenciam diretamente sua distribuição, podendo causar a aproximação ou o isolamento de populações.

Neste sentido, o rio Bonito apresenta um nítido desnível no relevo ao longo de seu percurso (variação de $541 \mathrm{~m}$ ), o que o caracteriza como um rio montanhoso, com presença de quedas d'água, as quais, aparentemente, atuam como barreiras limitando a migração de espécies para trechos mais altos do rio e promovem o endemismo. $\mathrm{O}$ valor de similaridade obtido entre o curso médio e baixo do rio Bonito indicou pouca semelhança entre as comunidades, uma vez que somente quatro espécies foram comuns entre estes dois ambientes.

Para Caramaschi (1986), a topografia é um fator determinante na distribuição longitudinal da ictiofauna, visto que em rios de relevo acidentado pode ocorrer a redução do número de espécies, ou 


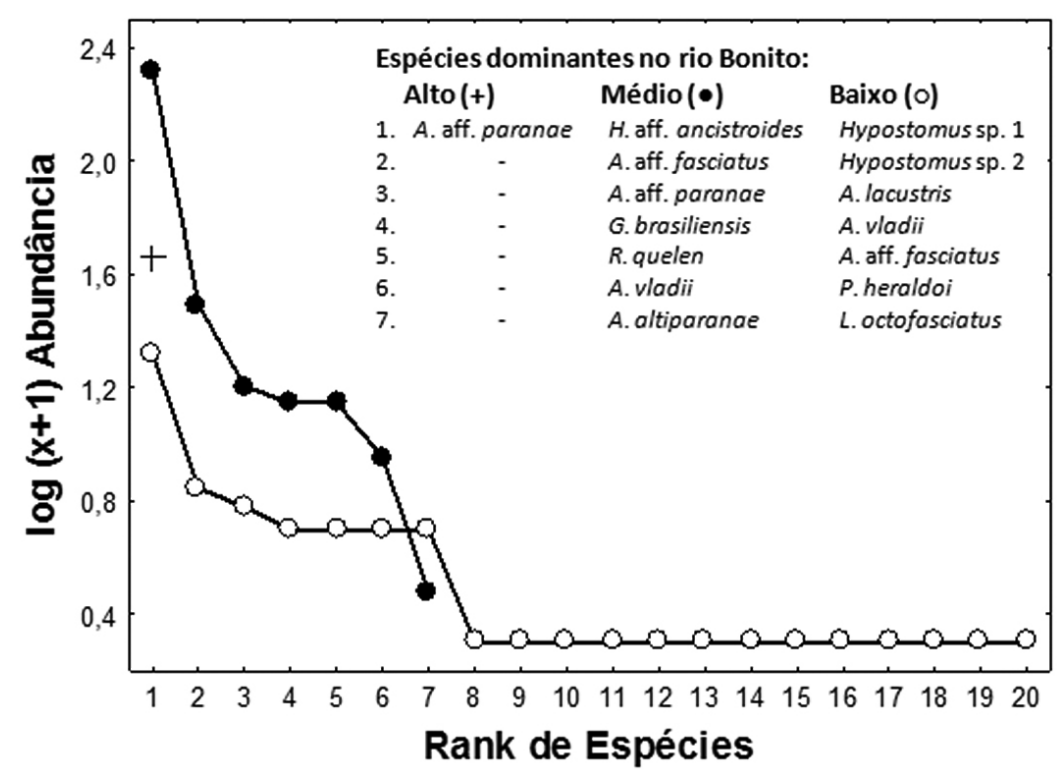

Figura 3. Curvas espécie-abundância das espécies coletadas nas unidades amostrais do rio Bonito (trechos alto, médio e baixo), afluente da margem esquerda do rio Ivaí, Estado do Paraná. A tabela no interior da figura lista em ordem decrescente as sete espécies mais abundantes nos trechos alto, médio e baixo do rio Bonito. As espécies raras do trecho baixo do rio Bonito ( $8^{\mathrm{a}}$ a $20^{\mathrm{a}}$ do rank) são: Apareiodon affinis, Astyanax sp., Brycon nattereri, Galeocharax knerii, Geophagus brasiliensis, Hypostomus ancistroides, Hypostomus albopunctatus, Imparfinis borodini, Leporinus amblyrhynchus, Oligosarcus paranensis, Pimelodus ornatus, Rhamdia quelen e Trichomycterus sp.

Figure 3. Species-abundance curves of the species collected in the sampling units of the Bonito river (upper, medium and lower stretches), a left margin tributary of the Ivaí river, State of Paraná. The table inside the figure lists, in descending order, the seven most abundant species in the upper, medium and lower stretches of the Bonito river. Rare species (ranked $8^{\text {th }}$ to $20^{\text {th }}$ ) of the lower stretches of the Bonito river are: Apareiodon affinis, Astyanax sp., Brycon nattereri, Galeocharax knerii, Geophagus brasiliensis, Hypostomus ancistroides, Hypostomus albopunctatus, Imparfinis borodini, Leporinus amblyrhynchus, Oligosarcus paranensis, Pimelodus ornatus, Rhamdia quelen, and Trichomycterus sp.

substituição por espécies congêneres. De acordo com Buckup (1999), a estrutura da ictiofauna de riachos é fortemente influenciada pelo gradiente altitudinal, o qual determina a velocidade de correnteza, estrutura do substrato e várias outras características ecológicas. Videla \& Bistoni (1999) verificaram uma associação da estrutura das comunidades de peixes com o gradiente de altitude no rio serrano de Los Sauces (Argentina). Estes autores constataram um incremento na riqueza e diversidade de espécies, bem como uma redução da dominância da cabeceira do rio em direção à sua foz. Os autores sugerem que essas diferenças devem estar associadas às mudanças nas condições abióticas ao longo do gradiente longitudinal do rio.

O perfil de predominância das ordens Siluriformes e Characiformes quanto à abundância, riqueza e biomassa no rio Bonito também tem sido verificado para o alto rio Paraná (Pelicice et al. 2005, Langeani et al. 2007, Esguícero \& Arcifa 2011), bem como para a ictiofauna neotropical (Lowe-Mcconnell 1999). De acordo com Castro (1999), a fauna de peixes de água doce continental no mundo é dominada tanto em termos de diversidade taxonômica como em biomassa por Ostariophysi, que compreendem aproximadamente $87 \%$ das espécies conhecidas, divididas primariamente entre as ordens Siluriformes com pelo menos 14 famílias e $47 \%$ das espécies e Characiformes, com pelo menos 15 famílias e cerca de $37 \%$ das espécies.

É notável a dominância de espécies de Loricariidae no rio Bonito $(70 \%$ da frequência relativa no trecho médio e $50 \%$ no baixo). Os loricarídeos constituem a maior família de Siluriformes com cerca de 550 espécies e com ampla distribuição na região neotropical, ocorrendo em uma grande variedade de ambientes de água doce (Buckup 1999). No presente trabalho, os maiores valores de abundância e de biomassa para as espécies desta família podem estar associados às características do rio, uma vez que todos os seus representantes são do gênero Hypostomus. Segundo Casatti et al. (2005), a presença de espécies deste gênero está associada a riachos com boa disponibilidade de corredeiras e boa qualidade de vegetação ripária. Parâmetros estes, observados nos trechos médio e baixo do rio Bonito, onde foram capturados os espécimes desse gênero.

A hipótese de que o rio Bonito apresenta características que favoreçam abundância de Hypostomus já foi levantada anteriormente por Viana et al. (2008). Estes autores analisaram a estrutura populacional de Hypostomus aff. ancistroides (Hypostomus cf. ancistroides naquele estudo) no trecho médio do rio Bonito e notaram que o fator de condição na espécie foi pouco influenciado pelo peso das gônadas, o que sugere boa disponibilidade de alimento nos ambientes amostrados e boa qualidade da vegetação ripária.

De acordo com o índice de constância de Dajoz (1983), a ictiofauna do rio Bonito é formada por espécies constantes e acessórias. Segundo Lemes \& Garutti (2002), a constância reflete a habilidade da espécie em explorar os recursos disponíveis no ambiente em um determinado período. O número de espécies constantes foi maior no trecho médio, seguido pelo trecho baixo, o que corrobora com a constatação de melhoria da qualidade de habitats nos trechos médio e baixo do rio Bonito. A presença de Astyanax aff. paranae no trecho superior pode não ser influenciada pelas condições de assoreamento observadas na cabeceira do rio. Ferreira (2004) estudou a ecologia e hábito alimentar de $A$. paranae em córregos do rio Passa Cinco, no Estado de São Paulo, onde a espécie apresentou preferência por itens alimentares alóctones e não sofreu nenhuma influência do assoreamento nestes riachos devido à entrada de recursos externos. A constância desta espécie capturada em ambiente bastante impactado pode, também, estar associada ao seu hábito alimentar.

A predominância de espécies acessórias no trecho inferior permite inferir que a ictiofauna dessa área seja compartilhada com o rio Ivaí, 
as quais podem visitar o trecho da foz do rio Bonito, mas utilizar o rio Ivaí na exploração da maior parte de seus nichos. Esta hipótese já foi considerada por Súarez \& Petrere Júnior (2006), comparando as riquezas de espécies na foz dos rios Iguatemi e Jogui. Eles atribuíram a maior riqueza de espécies na região da foz do rio Jogui, a um maior fluxo de espécies entre este e sua planície de inundação e a presença de espécies de ambientes lênticos, sob influência da menor velocidade da corrente da água verificada neste último. Apone et al. (2008) em um estudo da composição da ictiofauna do rio Quilombo, afluente do rio Mogi-Guaçú observaram uma diminuição no número de espécies constantes e aumento de espécies acidentais e acessórias a partir da cabeceira em direção a foz. Segundo os autores fatores como o decréscimo de altitude, aumento do volume de água, da largura e da profundidade, maior diversidade de micro-habitats e a maior proximidade ao rio Mogi-Guaçú, talvez proporcionem ambientes mais facilmente colonizáveis do que aqueles encontrados nas cabeceiras. Desta forma, muitas espécies vindas do rio MogiGuaçú podem ocupar os trechos inferiores do rio Quilombo com mais facilidade. A zona de contato entre a foz do rio Bonito e o rio Ivaí, ambientes com características estruturais e dinâmicas distintas, pode ser caracterizada como um ecótono. Nessa zona de transição as espécies podem coexistir, explorando seus habitats temporariamente, o que tende a aumentar a riqueza de espécies nessas áreas. Kolasa $\&$ Zalewski (1995) demonstram que os ecótonos exercem um papel crucial no estabelecimento dos padrões de diversidade de peixes.

Uma análise temporal poderia fornecer subsídios acerca do padrão de estabilidade da assembléia de peixes ao longo do ano, avaliando eventuais deslocamentos de espécies relacionados a eventos sazonais ou padrões de variabilidade natural na abundância. No entanto, é importante ressaltar que estudos desenvolvidos em riachos de cabeceira no alto rio Paraná têm revelado um padrão de baixa influência da sazonalidade na estruturação da assembléia de peixes, sendo as variações espaciais mais relevantes na distribuição para a maioria das espécies (Súarez 2008, Couto \& Aquino 2011).

$\mathrm{O}$ maior número de espécies constantes no médio rio Bonito também pode ser explicado pelas características fisiográficas desse trecho, com substrato rochoso e grande disponibilidade de microhabitats. A montante se encontra a cabeceira, supostamente com condições mais restritivas para a maioria das espécies e a jusante está uma cachoeira de 80 metros separando os trechos médio e baixo do rio Bonito. Portanto, as espécies estão atualmente isoladas neste trecho superior, o que favorece o desenvolvimento de melhores performances adaptativas (sensu Arnold 1983) em relação às condições do meio e, por isso, as espécies tendem a tornarem-se mais constantes. O gradiente elevado de montante para jusante confere uma fisionomia de corredeiras entremeadas por remansos, características estas que contribuem para o desenvolvimento de peixes reofílicos como os representantes de Hypostomus e Apareiodon, além de peixes de ambiente mais lênticos como Geophagus brasiliensis e as espécies do gênero Astyanax. O hábito alimentar onívoro dessas espécies também lhes favorecem pelo fato de explorarem uma grande variedade de itens alimentares. Lemes \& Garutti (2002) também atribuíram a constância de algumas espécies ao hábito alimentar onívoro em seu estudo em riachos da bacia do ribeirão Ouvidor.

O trecho inferior também revela indicativo de boas condições da sua vegetação ripária. Um desses indícios foi a ocorrência nesse trecho da espécie herbívora Brycon nattereri, por ser considerada sensível às degradações ambientais. Essa espécie é tida como rara e já foi até mesmo considerada extinta da ictiofauna paranaense (Shibatta et al. 2007), sendo um de seus últimos registros verificado no rio Tibagi (Gealh 2007). Portanto, por estar em risco de extinção, se faz necessário o estabelecimento de prioridades para sua proteção, bem como de seu habitat.
O processo de degradação ambiental dos rios causado pela ocupação desordenada das margens para atividades agrícolas e pecuárias é verificado, principalmente, no trecho superior do rio Bonito. Sabe-se que esse processo atua sobre a ictiofauna, interferindo na história natural das espécies, principalmente devido à modificação de seus micro-habitats. Adicionalmente, a escassez de inventários ictiofaunísticos na bacia do rio Ivaí também justifica o desenvolvimento de programas de pesquisas na região, na medida em que essa bacia esta sujeita às mais diversas pressões antrópicas como, por exemplo, a possibilidade da exploração de seu potencial hidrelétrico. Diante disto, é importante a continuidade dos estudos sobre a ictiofauna do rio Bonito por meio de um programa de monitoramento, buscando melhor compreensão acerca dos seus padrões ecológicos.

\section{Agradecimentos}

Somos gratos a Evanilde Benedito, Paulo R. da Silva e Rogério P. Mateus pela leitura crítica do manuscrito e valiosas sugestões; a Denis Lucas Viana pela ajuda na realização das coletas; a Rafaela L. Vendramel e Andreia Isaac pelo auxílio nos procedimentos laboratoriais; ao IBAMA pela licença de pesquisa científica concedida a CHZ (No 16053-1). Ao Nupélia/UEM e ao Programa de PósGraduação em Biologia Evolutiva (UNICENTRO/UEPG) pelo apoio logístico, ao Conselho de Desenvolvimento Científico e Tecnológico (CNPq) pelo apoio financeiro a CHZ (proc. 306066/2009-2) e à Coordenação de Aperfeiçoamento de Pessoal de Nível Superior (CAPES) pela bolsa concedida à HFV.

\section{Referências}

APONE, F., OLIVEIRA, K.A. \& GARAVELLO, C, J. 2008. Composição da ictiofauna do rio Quilombo, tributário do rio Mogi-Guaçu, bacia do alto rio Paraná, sudeste do Brasil. Biota Neotrop. 8 (1): http://www. biotaneotropica.org.br/v8n1/pt/abstract?article+bn02208012008.

ARAÚJO, B.N. \& TEJERINA-GARRO, L.F. 2007. Composição e diversidade em riachos do Cerrado, bacia do ribeirão Ouvidor, alto rio Paraná, Goiás, Brasil. Rev. Brasil. Zool. 24(4):981-990.

ARAÚJO, B.N. \& TEJERINA-GARRO, L.F. 2009. Influence of environmental variables and anthropogenic perturbations on stream fish assemblages, Upper Paraná River, Central Brazil. Neotrop. Ichthyol. 7(1):31-38. http:// dx.doi.org/10.1590/S1679-62252009000100005

ARAÚJO, M.I., DELARIVA, R.L., BONATO, K.O. \& SILVA, J.C. 2011. Fishes in first order stream in Ivaí River drainage basin, upper Paraná River Basin, Paraná state, Brazil. Check List 7(6):774-777.

ARNOLD, S.J. 1983. Morphology, performance and fitness. Amer. Zool. 23:347-361

BUCKUP, P.A. 1999. Sistemática e biogeografia de peixes de riachos. In Ecologia de peixes de riachos (E.P. Caramaschi, R. Mazzoni \& P.R. Peres-Neto, eds.). Série Oecologia Brasiliensis, Rio de Janeiro, p.91-138.

BUCKUP, P.A., MENEZES, N.A. \& GHAZZI, M.S. 2007. Catálogo das espécies de peixes de água doce do Brasil. Museu Nacional, Rio de Janeiro.

CARAMASHI, E.P. 1986. Distribuição da ictiofauna de riachos das Bacias do Tietê e do Paranapanema, junto ao divisor de águas (Botucatu-SP). Tese de Doutorado, Universidade Federal de São Carlos, São Carlos.

CASTRO, R.M.C. 1999. Evolução da ictiofauna de riachos sul americanos: padrões gerais e possíveis processos casuais. In Ecologia de peixes de riachos (E.P. Caramaschi, R. Mazzoni \& P.R. Peres-Neto, eds.). Série Oecologia Brasiliensis, Rio de Janeiro, p.139-155. 
CASTRO, R.M.C., CASATTI, L., SANTOS, H.F., FERREIRA, K.M., RIBEIRO, A.C., BENINE, R.C., DARDIS, G.Z.P., MELO, A.L.A., STOPIGLIA, R., ABREU, T.X., BOCKMANN, F.A., CARVALHO, M., GIBRAN, F.Z. \& LIMA, F.C.T. 2003. Estrutura e composição da ictiofauna de riachos do rio Paranapanema, sudeste e sul do Brasil. Biota Neotrop. 3(1): http://www.biotaneotropica.org.br/v3n1/ pt/ abstract?article+BN01703012003.

CASATTI, L., ROCHA, C.F. \& PEREIRA, C.D. 2005. Habitat use by two species of Hypostomus (Pisces, Loricariidae) in southeastern Brazilian streams. BiotaNeotrop. 5(2): http://www.biotaneotropica.org.br/v5n2/pt/ fullpaper?bn02905022005+en.

COUTO, T.B.A. \& AQUINO, P.D.U. 2011. Structure and integrity of fish assemblages in streams associated to conservation units in Central Brazil. Neotrop. Ichthyol. 9(2):445-454. http://dx.doi.org/10.1590/ S1679-62252011000200023.

DAJOZ, R. 1983. Ecologia geral. Vozes, São Paulo.

EIGENMANN, C.H. \& EIGENMANN, R.S. 1891. A catalogue of the fresh-water fishes of south America, United States National Museum, Washington.

ESGUÍCERO, A.L.H. \& ARCIFA, M.S. 2011. The fish fauna of the JacaréGuaçu River basin, Upper Paraná River basin. Biota Neotrop.11(1): http:// www.biotaneotropica.org.br/v11n1/en/abstract?article+bn 01811012011

FERREIRA, A. 2004. Ecologia trófica de Astyanax paranae (Osteichthyes, Characidae) em córregos da bacia do Rio Passa Cinco, estado de São Paulo. Dissertação de Mestrado, Universidade de São Paulo, São Paulo.

FOWLER, H.W. 1948. Os peixes de água doce do Brasil. Arq. Zool. São Paulo 6(1):1-204.

GALVES, W., SHIBATTA, O.A. \& JEREP, F.C. 2009. Estudos sobre diversidade de peixes da bacia do alto rio Paraná: uma revisão histórica. Semina: Ciênc. Biol. Saúde 3(2):141-154.

GÉRY, J. 1969. The freshwater fishes of South America. In Biogeography and Ecology in South America (E.J. Fittkau, J. Illies, H. Klinge, G. Shwabe \& H. Sioli, eds.). The Hague, W. Junk, v.2, p.828-848.

GEALH, A.M. 2007. Ictiofauna dos rios Fortaleza, Iapó, alto e médio Tibagi. In Patrimônio natural dos campos gerais do Paraná (M.S. Mello, R.S. Moro \& G.B. Guimarães, org.). UEPG, Ponta Grossa.

GRAÇA, W.J. \& PAVANELLI, C.S. 2007. Peixes da planície de inundação do alto rio Paraná e áreas adjacentes. Eduem, Maringá.

HUTCHESON, K. 1970. A test for comparing diversities based on the Shannon formula. J. Theoret. Biol. 29:151-154. http://dx.doi.org/10.1016/00225193(70)90124-4

KOLASA, J. \& ZALEWSKI, M. 1995. Notes on ecotone attributes and functions. In The importance of aquatic-terrestrial ecotones for freshwater fish (F. Schiemer, M. Zalewski \& J.E. Thorpe, eds). Kluwer Academic Publishers, Dordrecht. p.1-7. (Developments in Hydrobiology; 105).

LANGEANI, F., CASTRO, R.M.C., OYAKAWA, O.T., SHIBATTA, O.A., PAVANELli, C.S. \& CASATTI, L. 2007. Diversidade da ictiofauna do Alto Rio Paraná: composição atual e perspectivas futuras. Biota Neotrop. 7(3): http://www.biotaneotropica.org.br/v7n3/pt/ abstract?article+bn0340703.

LEMES, M.E. \& GARUTTI, V. 2002. Ecologia da ictiofauna de um córrego de cabeceira da bacia do alto Rio Paraná. Iheringia Série Zool. 92(3):69-78.

LOWE-McCONNELL, R.H. 1999. Estudos ecológicos de comunidades de peixes tropicais. EdUSP, São Paulo.
LUDWIG, J.A. \& REYNOLDS, J.F. 1988. Statistical ecology: a primer on methods and computing. Wiley-Interscience, New York.

LUNDBERG, J.G. 1993. African-South American freshwater fish clades and continental drift, problems with a paradigm. In Biological relationships between Africa and South America (P. Goldblatt, ed.). Yale University press, New Haven, p.156-199.

McALLISTER, D.E., HAMILTON, A.L. \& HARVEY, B. 1997. Global freshwater biodiversity: striving for the integrity of freshwater ecosystems. Sea Wind 11:1-140.

MAGURRAN, A. 1988. Ecological Diversity and its Measurement. Chapman \& Hall, London. http://dx.doi.org/10.1007/978-94-015-7358-0

MAIER, A., ZAWADZKI, C.H., DA GRAÇA, W.J. \& BIFI, A.G. 2008. Fish, Barra Bonita River, upper Paraná river basin, state of Paraná, Brazil. Check List 4(3):336-340.

PELICICE, F.M., AGOSTINHO, A.A. \& GOMES, L.C. 2005. Biodiversidade e conservação de peixes da planície de inundação do alto rio Paraná Cader. Biodiversid. 5(1):34-44.

REIS, R.E., KULLANDER S.O. \& FERRARIS JUNIOR, C. J. 2003. Check list of the freshwater fishes of South and Central America. Editora da Pontifícia Universidade Católica do Rio Grande do Sul, Porto Alegre, $729 \mathrm{p}$.

SCHAEFER, S.A. 1998. Conflict and resolution: impact of new taxa on phylogenetic studies of the neotropical cascudinhos (Siluroidei: Loricariidae). In Phylogeny and Classification of Neotropical Fishes (L.R Malabarba, R.E. Reis, R.P. Vari, Z.M.S. Lucena, \& C.A.S. Lucena, eds.). EdiPUCRS, Porto Alegre, Rio Grande do Sul, p.375-400.

SHIBATTA, O.A., GEALH, A.M. \& BENNEMANN, S.T. 2007. Ictiofauna dos trechos alto e médio da bacia do Rio Tibagi, Paraná, Brasil. Biota Neotrop. 7(2): http://www.biotaneotropica.org.br/v7n2/pt/ fullpaper?bn02107022007+pt.

SÚAREZ, Y.R. 2008. Spatial and temporal variation in fish species diversity and composition in streams of Ivinhema River basin, upper Paraná River. Biota Neotrop. 8(3): http://www.biotaneotropica.org.br/v8n3/en/ abstract?article+bn02308032008.

SÚAREZ, Y.R. \& PETRERE JUNIOR, M. 2006. Gradientes de diversidade nas comunidades de peixes da bacia do rio Iguatemi, Mato Grosso do Sul. Iheringia Sér. Zool. 96(2):197-204.

TEIXEIRA, T.P., PINTO, B.C.T., TERRA, B.F., ESTILIANO, E.O., GRACIA, D. \& ARAUJO, F.G. 2005. Diversidade das assembléias de peixes nas quatro unidades geográficas do rio Paraíba do Sul. Iheringia Série Zool. 95(4):347-357.

VANOTTE, R.L., MINSHALL, G.W., CUMMINS, K.W., SEDELL, J.R. \& CUSHING, C.E. 1980. The river continuun concept. Can. J. Fish. Aquat. Sci. 37:130-137. http://dx.doi.org/10.1139/f80-017

VIANA, D., WOLLF, L.L., ZALESKI, T., ROMÃO, S., BERTOLDI, G. \& DONATTI, L. 2008. Population Structure and Somatic Indexes of Hypostomus cf. ancistroides (Siluriformes, Loricariidae) collected from the Bonito River, Ivaí River Basin, Turvo, Paraná. Braz. Archiv. Biol. Technol. 51(3):493-502. http://dx.doi.org/10.1590/S151689132008000300008

VIDELA, M.M. \& BISTONI, A.M. 1999. Composición y estructura íctica de un rio serrano a lo largo de un gradiente altitudinal. Iheringia Série Zool. 87:171-180.

ZAR, J.H. 1999. Biostatistical Analysis. Prentice-Hall, New Jersey.

Recebido em 25/01/2012

Versão reformulada recebida em 20/04/2013

Publicado em 05/05/2013 


\section{Apêndice}

\section{Chave de identificação para as espécies de peixes do rio Bonito}

1. Linha lateral descontínua; nadadeira pélvica situada em posição torácica, isto é, sob as nadadeiras peitorais, maioria das escamas ctenóides Geophagus brasiliensis

- Linha lateral contínua; nadadeira pélvica situadas em posição abdominal, isto é, situada posteriormente a nadadeira peitoral; escamas ciclóides (quando presentes)

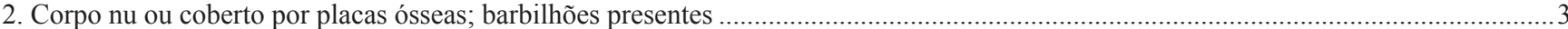

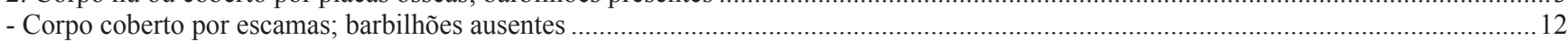

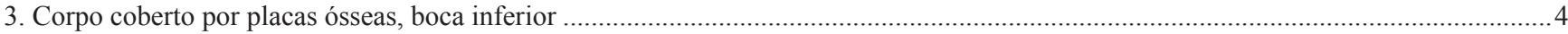

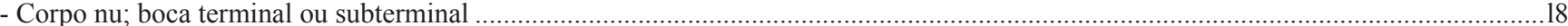

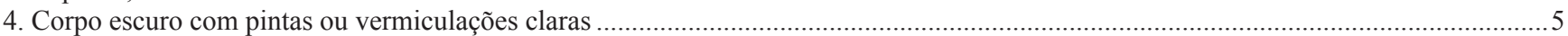



5. Nadadeira pélvica maior ou igual à nadadeira peitoral; 25 a 40 dentes robustos em cada hemi-série do pré-maxilar; abdômen amplamente coberto por placas diminutas ......................................................................................................................... Hypostomus albopunctatus - Nadadeira pélvica evidentemente menor do que a nadadeira peitoral; 40 a 70 dentes delgados em cada hemi-série do pré-maxiliar; abdômen parcialmente coberto por placas diminutas.

Hypostomus sp. 1

6. Cabeça deprimida; pintas da região posterior do corpo maiores do que o diâmetro orbital; abdômen parcialmente coberto por diminutas

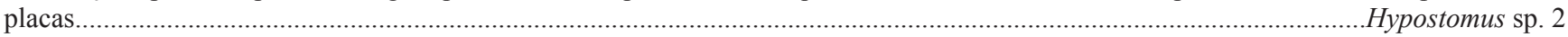

- Cabeça comprimida; pintas da região posterior do corpo menor ou igual ao diâmetro orbital; abdômen completamente coberto por diminutas placas..

7. Pintas escuras formando cinco a seis séries transversais sobre a nadadeira dorsal; nadadeira caudal furcada .....Hypostomus ancistroides

- Pintas escuras formando oito a dez séries transversais sobre a nadadeira dorsal; nadadeira caudal truncada (isto é, raios indivisos superior e inferior aproximadamente do mesmo tamanho dos raios ramificados).

8. Opérculo e interopérculo sem espinhos; barbilhões nasais ausentes Hypostomus aff. ancistroides

- Opérculo e interopérculo; barbilhões nasais presentes.

Trichomycterus sp.

9. Corpo baixo; altura de 9,5 a 10,5 vezes no comprimento padrão. Imparfinis borodini

- Corpo alto; altura de 3,0 a 5,5 vezes no comprimento padrão R................. 10

10. Raio indiviso da nadadeira dorsal não pungente.

- Raio indiviso da nadadeira dorsal pungente, isto é, em forma de espinho Rhamdia quelen

11. Corpo com uma faixa longitudinal clara sobre a cabeça e uma faixa escura na nadadeira dorsal.

- Corpo sem faixas e com várias séries longitudinais de manchas escuras arredondadas

12. Mandíbula de borda reta em vista ventral e sem dentes laterais.....

....................... 11

- Mandíbula de borda arredondada em vista ventral e com dentes laterais .....

..Pimelodus ornatus

.

13. Escamas pré-anais 29 ou mais; dentes do pré-maxilar com 12 ou mais cúspedes

- Escamas pré-anais 29 ou menos; dentes do pré-maxilar com menos de 12 cúspides Apareiodon affini

14. Dentes caninos Apareiodon vladii

Dentes incisivos, cuspidados ou molariformes

. .15

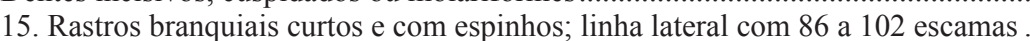

- Rastros branquiais longos e sem espinhos; linha lateral com 45 a 55 escamas.

16. Linha lateral bem abaixo do meio do corpo

Acestrorhynchus lacustris Oligosarcus paranensis

- Linha lateral em posição mediana do corpo Brycon natereri

- Duas séries de dentes no pré-maxilar e dentário; dentes tri ou pentacuspidados ....

18. Doze escamas circumpedunculares; uma faixa longitudinal escura sobre a linha lateral...

- Dezesseis escamas circumpedunculares; corpo com oito faixas transversais escuras .............................................Leporinus octofasciatus

19. Mancha umeral escura longitudinalmente ovalada e com limites bem definidos; maxilar sem dentes.....................Astyanax altiparanae

- Mancha umeral escura transversalmente alongada e com limites difusos; maxilar com um ou mais dentes ...........................................20

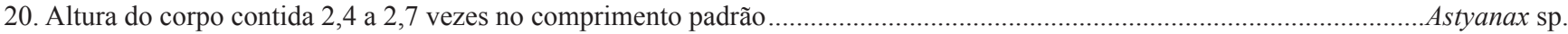

- Altura do corpo contida 2,9 a 3,6 vezes no comprimento padrão . .21

21. Nadadeira anal com 24 a 28 raios; linha lateral com 34 a 36 escamas Astyanax aff. fasciatus

- Nadadeira anal com 17 a 23 raios; linha lateral com 38 ou 39 escamas Astyanax aff. paranae

\section{Listagem de Material Testemunho}

Characiformes- Acestrorhynchidae- NUP 10646 Acestrorhynchus lacustris, 1 (171,4 mm CP); Anostomidae- NUP 10648 Leporinus amblyrhynchus, 4 (124,9-156,9 mm CP); NUP 10636 Leporinus octofasciatus, 2 (146,6-203,8 mm CP); Characidae - NUP 10746 Astyanax altiparanae, 1 (91,3 mm CP); NUP 5869 Astyanax aff. fasciatus, 22 (11,6-97,4 mm CP); NUP 5875 Astyanax aff. paranae, 1 (77,4 mm CP); Astyanax sp., 14 (44.4-76.6 CP); NUP 8534 Brycon nattereri, 1 (148,5 mm CP); NUP 10737 Galeocharax knerii, 1 (164,4 mm CP); NUP 10647 Oligosarcus paranensis, 1 (187,7 mm CP); Parodontidae- NUP 10645 Apareiodon affinis, 1 (108,3 mm CP); NUP 10449 Apareiodon vladii, 11 (91,4-105,4 mm CP). Perciformes - Cichlidae - NUP 5877 Geophagus brasiliensis, 2 (41,4-61,3 mm CP). Siluriformes- Heptapteridae- NUP 5871 Imparfinis borodini, 1 (132,5 mm CP); NUP 10838 Rhamdia quelen, 4 (151,7-156,9 mm CP); Loricariidae- NUP 3950 Hypostomus albopunctatus, 14 (30,7-38,5 CP); NUP 3950 Hypostomus ancistroides, 14 (3,7-38,5 CP); NUP 5873 Hypostomus aff. ancistroides, 14 (14,5163,9 mm CP); NUP10634 Hypostomus sp. 1, 3 (127,8-134,2 mm CP); NUP 10635 Hypostomus sp. 2, 1 (129,0 mm CP); Pimelodidae- NUP 10649 Pimelodus microstoma, 4 (128,1-157,0 mm CP); NUP 10747 Pimelodus ornatus, 1 (322,8 mm CP); Trichomycteridae- NUP 10800 Trichomycterus sp., $6(27,9-35,8 \mathrm{~mm} \mathrm{CP})$. 